stick with any swizzle stick, because there are no swizzle sticks. Consequently we see that if the swizzle stick and the gizzle gick are not identical, as Quine argues, then they are identical only qua swizzle stick. In either event, we have no good reason for claiming that gizzle gicks do not exist.

University of Waterloo,

(C) W. R. Aввотt 1983

Waterloo, Ontario,

Canada N2L $3 G 1$

\title{
REFERENCES
}

[1] W. V. O. Quine, 'Identity, Ostension, and Hypostasis', in From a Logical Point of View, New York: Harper, 1963.

[2] W. V. O. Quine, 'What Price Bivalence', The Journal of Philosophy, LXXVIII (2), Feb. 1981.

[3] P. Grim, 'What Won't Escape Sorites Arguments', Analysis, 42.1 (January 1982), 38-43.

[4] P. Unger, 'There Are No Ordinary Things', Synthese, 41 (1979), 117-54.

[5] P. Unger, 'Scepticism and Nihilism', Nous, 14 (1980), 517-45.

\section{IS THIS A SWIZZLE STICK WHICH I SEE BEFORE ME?}

\section{By PATrick Grim}

Q

UINE AND UNGER take sorites arguments seriously against the existence of objects as normal as swizzle sticks, but both suggest precise replacements on the order of ' 1 -billion-atom swizzle sticks' ([3], [4]). W. R. Abbott [1] agrees with me that at least these will not do.

But what of more carefully constructed precise replacements such as gizzle gicks? With a few hedges these are defined as configurations of plastic molecules within a 100 molecule range of the closest physical approximation to a perfect $5^{\prime \prime}$ swizzloid, a shape patterned on that of an imagined paradigm swizzle stick.

Might not gizzle gicks escape sorites arguments? No - or so I originally argued, We start with the existential supposition

(9) There is at least one gizzle gick,

and note that this is inconsistent with:

(10) If anything is a gizzle gick, that is sufficient for it to be a swizzle stick, in the ordinary sense of the term. 
(11) If anything is a swizzle stick, in the ordinary sense of the term, then it consists of more than one atom, but only a finite number.

(12) If anything is a swizzle stick, in the ordinary sense of the term, then the net removal from it of one atom, or only a few, in a manner most innocuous and favourable, will not mean the difference as to whether or not there is a swizzle stick there, in the ordinary sense of the term ([2], p. 42).

(10), I argued, rests on linguistic intuititions at least as compelling as those behind Unger's original premises, represented here as (11) and (12). But if we are to maintain (10) through (12) within the bounds of consistency we must also deny (9); taking sorites arguments seriously, there are no gizzle gicks, either.

It is (10) that Abbott attacks.

Suppose that you were to put a gizzle gick, suitably defined, before me now. It's plastic, remember, and quite nearly swizzloid the ideal shape of an imagined paradigm swizzle stick. It's also blue, perhaps, or a peculiar shade of fuscia, and perfect for stirring bloody marys.

Is this a swizzle stick which I see before me?

'Yes', I say - or at least so I said originally.

'No', says Abbott. Gizzle gicks and swizzle sticks have different identity conditions. A swizzle stick can shed atoms and remain a swizzle stick, but a gizzle gick that loses one atom too many is no longer a gizzle gick. Gizzle gicks are thus not identical to swizzle sticks, and so (10) is false - that this is a gizzle gick is not sufficient for it to be a swizzle stick in the ordinary sense of the term.

I have thee not, and yet I see thee still.

Abbott's argument introduces a range of questions regarding identity and existence, and I think his treatment of these is wrong in a number of respects. I also consider these deep and difficult issues, however, and cannot hope to address them adequately here.

Luckily, I don't have to. For identity is simply a red herring - it is not in fact at issue, and the argument can easily be phrased without it.

Unger avoids similar complications of identity by phrasing his sorites arguments without it - (12) above, for example, maintains only that there will be $a$ swizzle stick after the removal of an atom, not that the same swizzle stick will survive (see also Unger's discussion of avoiding identity in [4], pp. 118-21). Here we can follow suit.

We do not in fact need (10) in the form that Abbott attacks. We need only $\left(10^{\prime}\right)$ :

$\left(10^{\prime}\right)$ If anything is a gizzle gick, that is sufficient for there to be a swizzle stick, in the ordinary sense of the term.

No gizzle gick, then, need be a swizzle stick. All we need is this: that were I to put a gizzle gick before you now, there would be 
something before you that would qualify as a swizzle stick in the ordinary sense of the term. With an eye to the definition of gizzle gick, and an appreciation for ordinary notions of swizzle sticks, I don't think that can plausibly be denied.

$\left(10^{\prime}\right)$ still, of course, rests on linguistic intuitions. But these intuitions seem at least as firm as those behind (11) and (12), borrowed from Unger's original. Issues of identity of the sort Abbott raises don't touch $\left(10^{\prime}\right)$ at all.

The argument above with $\left(10^{\prime}\right)$ substituted for (10) has the same force as before. If we are to maintain $\left(10^{\prime}\right)$ through (12) within the bounds of consistency, we must also deny (9); there are no gizzle gicks, either.

If we are to take sorites arguments seriously, then, even precise replacements of this sort will fall victim. And identity considerations such as Abbott's will not be enough to save them.

State University of New York,

(C) PATRICK GRIM 1983

Stony Brook, N.Y. 11794, U.S.A.

\title{
REFERENCES
}

[1] W. R. Abbott, 'A Note on Grim's Sorites Argument', Analysis, 43.4 (October 1983), 163-5.

[2] P. Grim, 'What Won't Escape Sorites Arguments', Analysis, 42.1 (January 1982), $38-43$.

[3] W. V. Quine, 'What Price Bivalence?', Journal of Philosophy, 77 (1981), 90-5.

[4] P. Unger, 'There Are No Ordinary Things', Synthese, 41 (1979), 117-54.

\section{AYER'S DEFINITION OF EMPIRICAL SIGNIFICANCE REVISITED}

\section{By M. L. PoKriEfKA}

\begin{abstract}
AYER'S definition of empirical significance is open to decisive Aobjections by Hempel, Church, Scheffler and Ullian. The definition can be repaired so as to evade these and similar objections. This may come as a surprise to those who believe, with Scheffler, that "no further restrictions on additional premises for definitions of the Ayer type can reasonably be expected to rule out all conjunctions with nonsensical components' $(7$, p. 155). Before offering a definition that does rule out all such conjunctions, it will be helpful to review Ayer's definition, some objections, some revisions of the definition, and some more objections.
\end{abstract}

